\title{
rBAN: retro-biosynthetic analysis of nonribosomal peptides
}

\author{
Emma Ricart ${ }^{1,2^{*}}$,, Valérie Leclère ${ }^{3}$, Areski Flissi ${ }^{4,5}$, Markus Mueller ${ }^{6}$, Maude Pupin $^{4,5}$ and Frédérique Lisacek ${ }^{1,2,7}$ (D)
}

\begin{abstract}
Proteinogenic and non-proteinogenic amino acids, fatty acids or glycans are some of the main building blocks of nonribsosomal peptides (NRPs) and as such may give insight into the origin, biosynthesis and bioactivities of their constitutive peptides. Hence, the structural representation of NRPs using monomers provides a biologically interesting skeleton of these secondary metabolites. Databases dedicated to NRPs such as Norine, already integrate monomer-based annotations in order to facilitate the development of structural analysis tools. In this paper, we present rBAN (retrobiosynthetic analysis of nonribosomal peptides), a new computational tool designed to predict the monomeric graph of NRPs from their atomic structure in SMILES format. This prediction is achieved through the "in silico" fragmentation of a chemical structure and matching the resulting fragments against the monomers of Norine for identification. Structures containing monomers not yet recorded in Norine, are processed in a "discovery mode" that uses the RESTful service from PubChem to search the unidentified substructures and suggest new monomers. rBAN was integrated in a pipeline for the curation of Norine data in which it was used to check the correspondence between the monomeric graphs annotated in Norine and SMILES-predicted graphs. The process concluded with the validation of the $97.26 \%$ of the records in Norine, a two-fold extension of its SMILES data and the introduction of 11 new monomers suggested in the discovery mode. The accuracy, robustness and high-performance of rBAN were demonstrated in benchmarking it against other tools with the same functionality: Smiles2Monomers and GRAPE.
\end{abstract}

Keywords: Peptide, Monomer, Retro-biosynthesis, Fragmentation, Structure analysis, Natural product, Curation, Substructure search

\section{Introduction}

Natural products are a well-recognized source for drug discovery due to their wide range of antibiotic, antitumor or immunosuppressant activities. Indeed, $26 \%$ of the drugs approved by the US FDA from 1981 to 2014 were natural products or natural products derivatives [1]. An important part of those are nonribosomal peptides (NRPs) considered as secondary metabolites and found in bacteria and fungi. In these organisms, NRPs are assembled by large enzymatic systems into complex structures from building blocks such as non-proteinogenic amino acids, fatty acids or carbohydrates. Significant portions of the bacterial and fungal genome are devoted to

\footnotetext{
*Correspondence: Emma.Ricart@sib.swiss

1 Proteome Informatics Group, SIB Swiss Institute of Bioinformatics, CMU,

Rue Michel-Servet 1, 1211 Geneva, Switzerland

Full list of author information is available at the end of the article
}

the production of these compounds. Therefore, genome mining tools such as GARLIC [2] and antiSMASH [3] have been developed to automatically identify secondary metabolite biosynthesis gene clusters. However, these tools are not able to distinguish between clusters of already known compounds and clusters uncovering new natural products. A possible approach to solve this problem is to perform the retro-biosynthesis of these compounds obtaining their constituent monomers and align them with the monomers of the predicted clusters $[2,4$, 5]. A few methods predicting the retrosynthesis of a compound from its chemical structure have been described. To begin with, CHUCKLES [6] can convert a chemical structure into a monomer-based sequence by matching a set of monomers against the target structure. The monomers are previously sorted by descending size and the matching is done sequentially. The main limitations of this method are: (i) larger monomers are given the

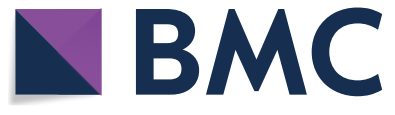

(c) The Author(s) 2019. This article is distributed under the terms of the Creative Commons Attribution 4.0 International License (http://creativecommons.org/licenses/by/4.0/), which permits unrestricted use, distribution, and reproduction in any medium, provided you give appropriate credit to the original author(s) and the source, provide a link to the Creative Commons license, and indicate if changes were made. The Creative Commons Public Domain Dedication waiver (http://creativecommons.org/ publicdomain/zero/1.0/) applies to the data made available in this article, unless otherwise stated. 
priority and (ii) monomers with more than three external connections are not handled. This approach is efficient with regular peptides, but not for NRPs. Other methods such as RECAP (Retrosynthetic Combinatorial Analysis Procedure) [7], BRICS (Breaking retrosynthetically interesting chemical substructures) [8] or molBLOCKS [9] use fragmentation rules to obtain drug-like chemical entities. However, these methods are focused on the discovery of structural motifs for drug design and they make no attempt to annotate the target compounds by identifying the resulting fragments. Moreover, their fragmentation rules are derived from common chemical reactions, lacking specificity for particular compounds such as NRPs.

In recent years, two new tools specifically designed to target NRPs have been published. The first one, Smiles2Monomers (s2m) [10] maps the monomers of a database within an atomic structure and selects the best combination (tiling) that covers the whole molecule with nonoverlapping monomers. This approach is algorithmically elegant but computationally expensive. As a result, the best tiling is obtained as an approximate solution and the optimal mapping is not always found, sometimes leading to uncovered regions in the molecule. A second solution is implemented in GRAPE (Generalized Retro-biosynthetic Assembly Prediction Engine) [2] as the theoretical deconstruction of NRPs and Polyketides (PKs) by applying specific retro-biosynthetic reactions. The obtained fragments are then matched against a monomer library integrated in the software. A sequence of monomers is given as a result, but the original monomer linkages are lost. Both, GRAPE and s2m rely on their monomer database, which is a limitation for the analysis of peptides containing new monomers.

Part of the interest in developing retro-biosynthesis tools arises from the benefit of a monomeric representation. Chemical structure databases dedicate an important part of their resources in data curation, analysis and visualization. The complex structure of NRPs often results in too dense and unclear atomic representations. A monomeric format, as with peptide sequence annotation, reduces the complexity of the layout providing the same information in a more intelligible way and facilitates the implementation of substructure and similarity search algorithms [11, 12]. Furthermore, this format is biologically meaningful as the monomers provide direct insights into the peptide activity and origin [11, 13, 14]. These substructures bring essential information to understand the biosynthesis of the peptide and, given their bioactivities, they are interesting data for structure-based drug design studies.

The convenience of the monomeric method is reflected in the emergence of new monomer-based notation formats. Examples of that are the recent languages named
HELM (Hierarchical Editing Language for Macromolecules) $[15,16]$ and SCSR (Self-Contained Sequence Representation) [17], which provide concise annotation of complex biopolymers in a component-based approach. Some databases devoted to bioactive peptides have also chosen this format to represent their data. This is the case of Norine [18, 19], which is entirely dedicated to NRPs and uses monomer graphs for structure depiction and analysis. Indeed, all the structural analysis tools integrated in Norine are monomer-based [10-12, 14], proving the advantages of the approach. Another example is the BIRD (Biologically Interesting molecule Reference Dictionary) [20] project from PDB (Protein Data Bank) [21]. Here, the peptide-like inhibitor and antibiotic molecules are represented as polymers with sequence information or as single components. BIRD is the result of a remediation work in which part of the PDB entries were reviewed in order to improve their representation and facilitate their identification and analysis. This kind of processes require a long and tedious effort that could be accelerated using bioinformatics tools. Hence, the usage of retro-biosynthesis software is decisive to improve these curation tasks by providing automatic annotation and assuring conciseness between the atomic and monomeric annotations. Additionally, the "in silico" retro-biosynthesis can also be applied to validate already annotated entries by checking the correspondence between the existing and the predicted annotations. A practice that would also spot potentially erroneous entries.

In this article, we introduce rBAN, a new tool simulating the retro-biosynthesis of NRPs. The main strategy of the software is to perform the fragmentation of a molecule by breaking it through a set of pattern bonds and matching the resulting fragments to a monomer database (Fig. 1). The matching process was specifically designed to allow tautomer's identification, a feature that was already presented in the $\mathrm{s} 2 \mathrm{~m}$ tool and named light matching. However, the two approaches are slightly different: the light matching of $\mathrm{s} 2 \mathrm{~m}$ omits all the implicit hydrogens and bond orders to match the monomer, while rBAN only omits the position of the double/triple bonds, making the method more restrictive and decreasing the likelihood of obtaining false positives. rBAN also introduces the "discovery mode" option that is applied when a monomer cannot be matched in the custom database. In this mode, missing substructure(s) can be automatically searched in PubChem [22] to suggest a new monomer. This feature reduces the dependence to the database, providing more flexibility than the retro-biosynthetic approaches previously presented. Finally, the results are presented in a directed graph format that includes the bond types linking the monomers. To our knowledge, no 


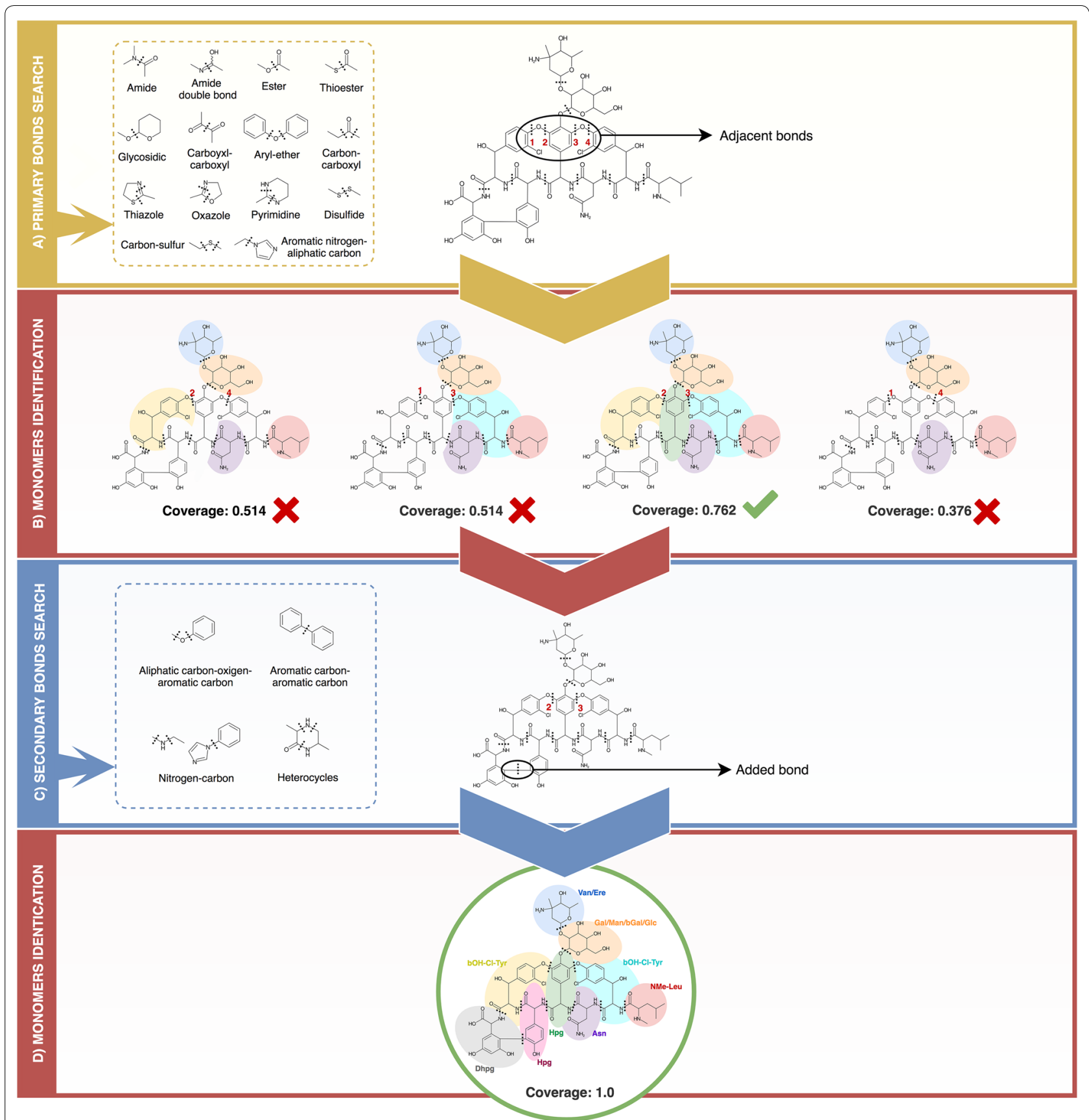

Fig. 1 Example of Vancomycin processing. A First, the primary bonds mapping searches the most common bonds between NRP monomers within the molecule. This process results in the mapping of two pairs of adjacent bonds that cannot be targeted simultaneously since it would isolate some atoms. To avoid that all the possible combinations only including one of the neighboring bonds are computed. B Then, rBAN retrieves the substructures resulting from each combination and it matches them against the monomer database. A coverage score is given to each combination based on the number of atoms that could be annotated. $\mathbf{C}$ In this case, any of the results has a full coverage, so the algorithm proceeds to the secondary bonds search of the structure with the highest score. D The breakage of a carbon-carbon bond results in the full mapping of the peptide

other tool provides the bond type annotation though it can be highly relevant for its integration into structural analysis pipelines. rBAN is presented in two formats, as an executable jar and as a web application, the latter being a simplified version of the software. We used rBAN 
for the curation of the Norine database and benchmarked it against $\mathrm{s} 2 \mathrm{~m}$ and GRAPE.

\section{Methods}

rBAN was developed in Java using the Chemistry Development Kit (CDK) library. Given an input file with chemical structures in SMILES (Simplified molecular-input line-entry system) [23], the tool uses CDK to map the target bonds by substructure search and Norine monomer database to identify the corresponding monomers. The overall process of the software architecture is described in the Fig. 2.

\section{Data preprocessing \\ NRPs monomer data}

Norine is dedicated to NRPs and was used to retrieve the monomer dataset in order to guarantee consistency between the target compounds and their building blocks. This dataset consists of 534 manually-annotated monomers extracted from the compositions of the NRPs in the database. Hence, the dataset is limited to the monomers present in Norine peptides and it may not be sufficient when used for the identification of fragments of new NRPs. To solve this issue, we developed an algorithm that suggests new monomers by adding modifications to the existing ones. In order to add a biological value to the predicted structures, the modifications were selected in accordance to some of the enzymatic reactions occurring in the NRP biosynthesis [24, 25] (see Additional file 1: Table S1). For instance, a methyl group is added in the amino side of each monomer in order to mimic the action of the methyltransferase (MT) domain. Finally, preprocessing is also used to identify monomers with identical chemical graphs (isomers) and group them as a single entry (the tool does not include isomer discrimination). The PubChem PUG-REST service is used to include the PubChem IDs of the monomers.

\section{Software architecture}

1. Primary bond search NRP monomers are usually connected through certain types of bonds, the most common being amino and ester. Therefore, mapping these bonds is the first step of monomer identification. We rely on a graph isomorphism algorithm provided by CDK to search the substructures of the bonds within the chemical graph of the target compound. The complete list of bond types included in the search (Fig. 1A) was manually constructed based on observations and literature [25-27]. Smiles Arbitrary Target Specification (SMARTS) [28] is the language used to describe the molecular patterns of the bonds since it provides higher flexibility than

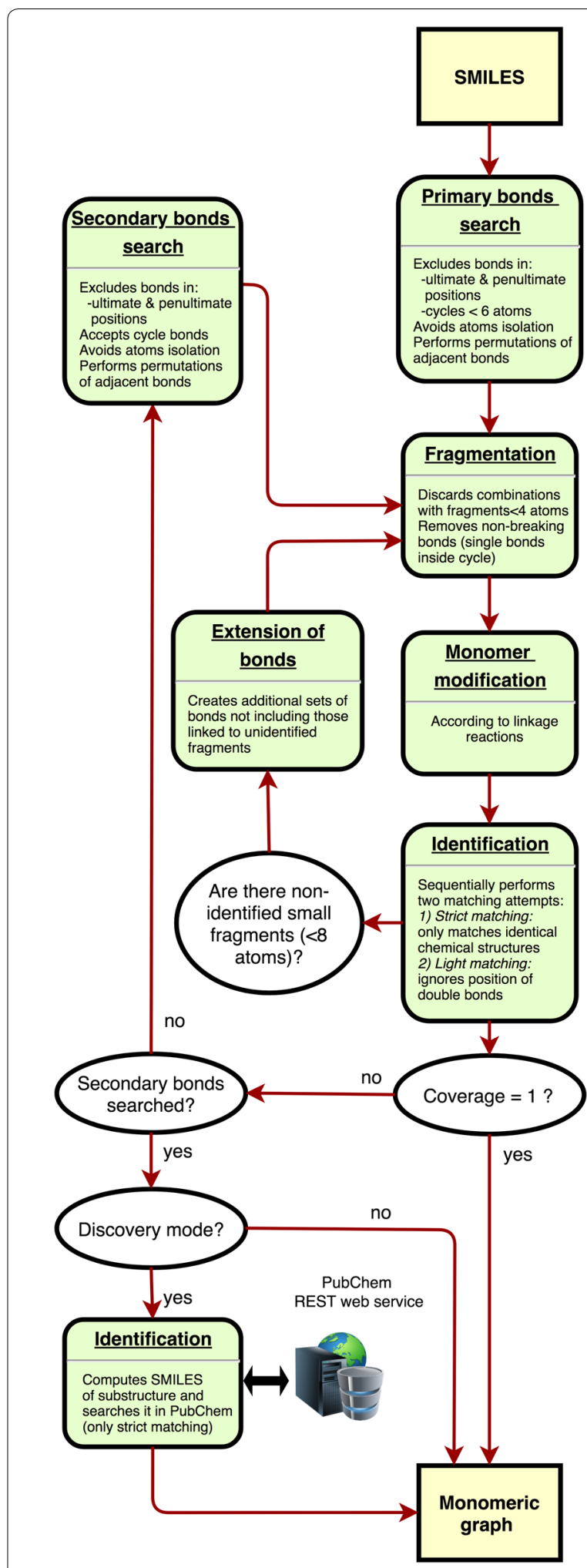

Fig. 2 Software architecture workflow. This flowchart describes the series of steps for processing structures with rBAN 
SMILES. During this step, all the bonds matching the target patterns will be selected unless they are positioned on terminal branches of the chemical structure (ultimate or penultimate positions). These bonds are excluded in order to avoid single atom isolation. Bonds pertaining to cycles of less than six atoms are also removed, although they will be evaluated later in the pipeline. The only exception to that rule are the oxazole and thiazole heterocycles, as they are highly abundant in NRPs [29].

Once mapped, the bonds between adjacent positions cannot be simultaneously targeted (single atom isolation problem). This issue is solved by computing multiple combinations, each combination only including one of the neighboring bonds. To do so, the adjacent bonds are grouped in different sets and a recursive algorithm computes the Cartesian product of these sets to generate all possible permutations. Note that to reduce the number of combinations and maximize the number of targeted bonds not all the adjacent bonds are included in this calculation, but only those whose simultaneous breakage implies the isolation of single atoms or pairs (Fig. 3). In a similar way, the presence of an amino or an ester bond in the set also limits combinatorics as they are prioritized due to their predominance as NRP links.

2. Fragmentation and identification The bonds mapped in the primary search are used as breaking points to obtain the fragments of the molecule. This is done using a breadth-first search algorithm to iterate through the chemical graph and compute the resulting fragments from those breakages. This action is performed for each permutation of bonds pro- vided, producing several sets of fragments that will be matched against the monomer database. Prior to this matching, the fragments are slightly modified in order to compute their expected structure outside the molecule -when not linked- thereby generating structures equivalent to those stored in the monomer database. The modifications applied are in accordance with the linkage patterns observed for each type of bond. For instance, a hydroxyl group is added to the formyl-ended fragment derived from a peptide bond breakage in order to obtain the "original" carboxyl-terminus structure of the monomer (see Additional file 1: Table S2). Once these modifications are applied, the monomers are matched against the database in order to identify them. Two different matching attempts are sequentially executed: the strict and the light matching. The strict matching will be only successful if the graph of the fragment is identical to the graph from the database. It checks the atom connectivities, the atom types and the bond orders. If a structure cannot be "strictly" matched, rBAN proceeds to light matching, which allows changes in the position of the double/triple bonds facilitating tautomer identification. Failure to match fragments can be due to the fragmentation of inner bonds in a monomer. Hence, when a fragment is not identified, the algorithm repeats the matching process by removing each of its linking bonds consecutively (Fig. 4). This process is limited to small-medium fragments (less than 8 atoms) because of their higher chances of being part of a monomer; such restriction also avoids an excessive amount of combinations. When a whole set of fragments has been matched, it is assigned with a score indicating the number of annotated atoms

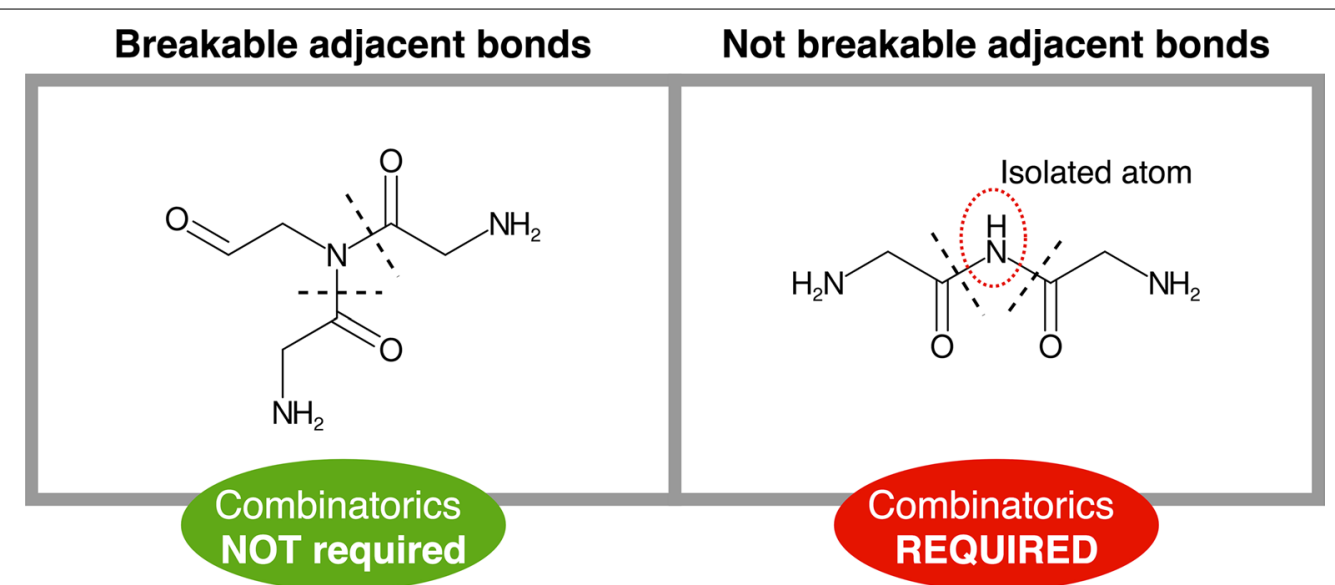

Fig. 3 Adjacent bonds breakage. Our fragmentation algorithm avoids atom isolation, which restricts the simultaneous cut of some adjacent bonds, requiring the computation of further combinations 
1. Bonds search - the primary bonds are mapped in the molecule

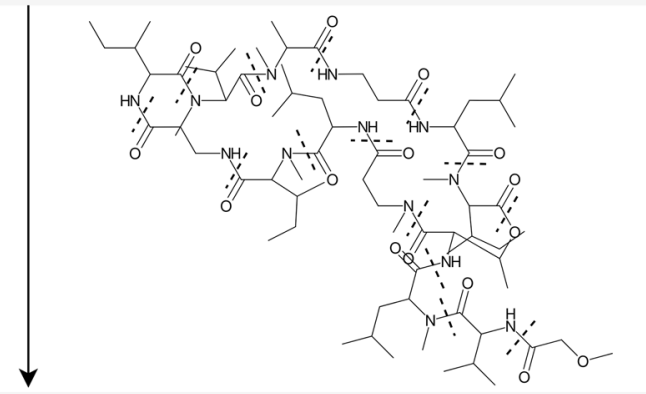

2. Monomers identification - the molecule is NOT fully annotated

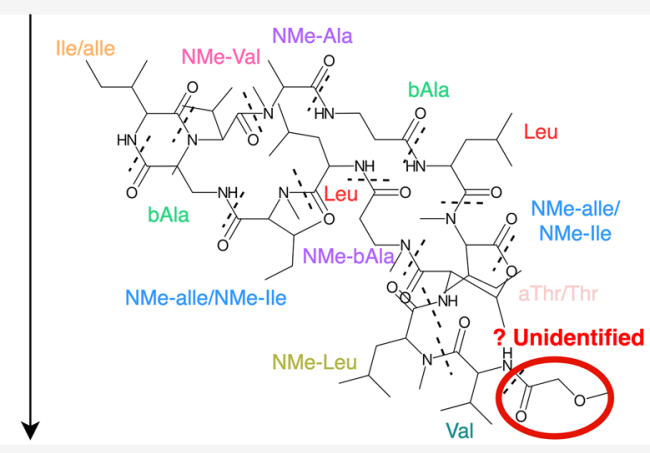

3. Removal of the bond linking the unidentified monomer

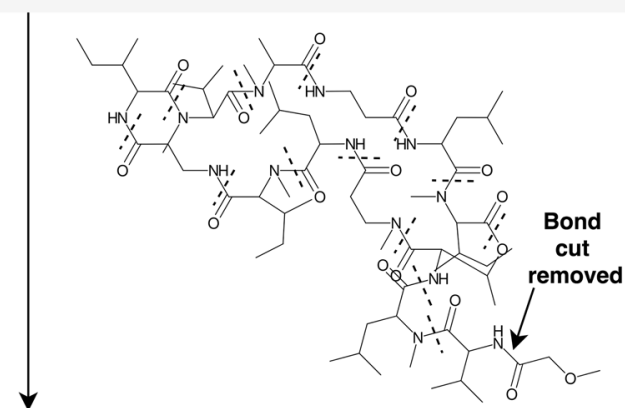

4. Monomers identification - the molecule is fully annotated

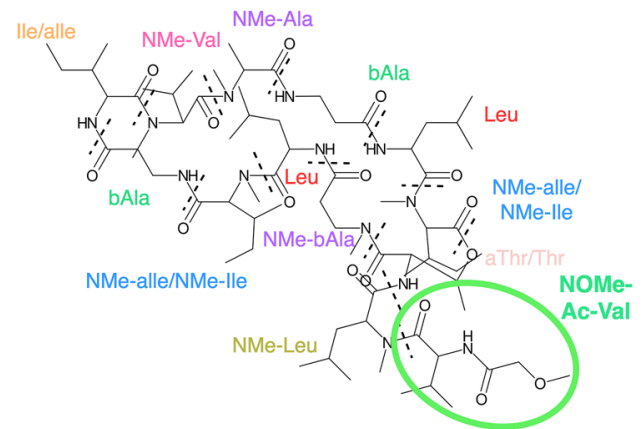

Fig. 4 Identification of monomers containing inner bonds. Some monomer bonds are sometimes fragmented by the algorithm. To handle these cases, when a small region cannot be identified, rBAN repeats the matching process after removing the bond linked to the unidentified substructure (example with Theonellapeptolide le) versus the total number of atoms in the molecule (coverage). The next steps in the pipeline depend on these scores. If any of the fragment sets has a score of 1, ergo all the monomers have been identified, the algorithm proceeds to the monomer graph creation. Otherwise, the secondary bonds search is applied to the sets with the highest score (Fig. 1B).

3. Secondary bond search Some bond types such as the carbon-carbon linkages are not common as a bridge between NRP monomers and breaking them in the initial step would lead to unnecessary and excessive fragmentation. This is why they are considered as secondary bonds and their mapping is restricted to the fragments that have not been identified. The secondary bond collection comprises less common bonds and non-specific heterocycles (Fig. 1C). Specific heterocycles such as the oxazoles and thiazoles are covered in the primary search, since their cyclisation patterns are well-known [27, 30]. Yet the existence of a wide range of cyclisation forms complicates the individual targeting of the remaining heterocycles. For this reason, we use a general approach that provides several breakage possibilities instead of a single solution. The algorithm performing this task implements substructure search to identify the heterocycles and combinatorics to return the permutations of cycle bonds that break the fragment without leaving isolated atoms. After the secondary bond search, the fragmentation and identification step is repeated. If the full score is still not reached and the monomer discovery mode is activated, rBAN moves to the next step.

4. Monomer discovery The unidentified substructures may represent missing monomers in the database. In these cases, the CDK library is used to generate the SMILES of the unknown chemical structure that serves as a parameter for an automatic PubChem search. The substructures successfully identified are annotated using their PubChem name and suggested in the results as new monomers for the Norine database. The information is presented in a JSON file where the compounds containing the suggested monomer are also listed. Graphical results are also provided. For each new monomer, rBAN creates a folder with the depictions of the peptides where the substructure occurs.

5. Monomer graph serialization The monomeric structure consists of a directed graph with a set of nodes represented by the predicted fragments and a set of edges symbolizing their linking bonds. To build this graph, the monomers are reconnected using the association between the broken bonds and the resulting fragments. The edges are labeled specify- 
ing the type of bond and their direction is chosen based on the type of atoms in the bond. The monomer associated with the carbon atom of the bond is set as the source while the monomer containing the heteroatom is set as the target. For instance, in case of a peptide bond, the monomer with the carboxylic side would be the source while the monomer with the amino side, the target. The graph is serialized in a JSON file also containing the atomic graph of the peptide that associates each atom with the monomer containing it (see Additional file 2). If the theoretical annotation (Norine graph) is given as an input, the output graph will also contain a "correctness" value. This value results from the division of the number of correctly annotated atoms (associated with the expected monomer) by the total number of atoms in the molecule. The graphical depiction of the chemical structures with the labeled monomers is also implemented as an option.

\section{Results and discussion}

The Norine database provides structural data of NRPs in both atomic and monomeric formats. The monomer annotation is essential to obtain the correctness of rBAN predictions and for this reason Norine was chosen as the main resource to evaluate the software.

\section{Norine Database Curation and Extension}

In Norine, the SMILES (atomic structure) and the monomer graphs (monomeric structure) are sometimes extracted from different resources. To guarantee the conciseness between the two representations and thereby validating the SMILES from Norine, rBAN was run to compare the SMILES-predicted monomeric graph with the Norine annotated graph. When the theoretical and the predicted graphs are identical, then the result is considered as correct and the corresponding SMILES is validated. From the 256 peptides that are described in SMILES, rBAN could validate 249 (97.26\%) (Fig. 5a1). The non-validated peptides were manually inspected and errors in their SMILES were identified. Hence, the lack of validation was attributed to wrong input data and not to a wrong mapping of the software. In fact, the software helped to spot these wrong SMILES that were later corrected/removed from the database. An example is Enniatin F, whose monomeric annotation did not match the structure given by the SMILES (Fig. 5b).

As already mentioned, in the previous version of Norine only 256 entries ( $21.56 \%$ of the total) contained the structural information in the SMILES format. In order to increase this count, we used the PubChem PUG-REST
Service to perform automatic searches, retrieve the missing SMILES and validate them using rBAN. The only available parameters for the PubChem searches were the name of the compound, which lacks specificity, and the PubChem link provided by Norine, that is rarely present and occasionally wrong. Hence, the validation step becomes essential to reduce the uncertainty of the search and provide more reliable results. From the 403 SMILES retrieved from PubChem, 242 were validated using rBAN (Fig. 5a2). These SMILES were added to the database generating a two-fold increase in Norine SMILES data. The non-validated entries were considered as false positives due to the uncertainty of the search.

\section{Monomers discovery}

As already mentioned, the non-validated entries can be due to a wrong annotation either in the SMILES or in the monomeric graph. In the latter case, peptides may contain monomers not present or wrongly annotated in Norine. Thus, rBAN was run in discovery mode to identify new monomers. The software suggested 61 new building blocks. Some of these predictions could be wrong due to mistakes in the input SMILES or wrong mapping of the software. Hence, a manual inspection was required before their addition into the database. To increase confidence, only the monomers present in more than one compound were evaluated.

From the 18 monomers examined, eleven were correct suggestions (Table 1 ). $N$-Formyl-Lysine was the most commonly found monomer, missing in Norine because $\mathrm{CO}$ is currently defined as a monomer in the database and occurs in several NRP graphs. In contrast, rBAN considers $\mathrm{CO}$ as formylation and not a monomer therefore suggested a new formylated monomer. Most of the other new entities correspond to monomers that were not properly annotated in the monomeric graph. Such is the case of the "C4:1(3)-OH(2)" monomer that should be beta-Vinyllactic acid (C5:1(4)-OH(2)) (see Additional file 1: Fig. S1). Other cases encompass a missing monomer in the monomeric graph or an incorrect SMILES of the known monomer. All the corrections were made in accordance to the literature associated with the corresponding compounds.

Seven of the monomers suggested by rBAN were rejected (find them in Additional file 1: Fig. S3) because the manual inspection of their corresponding peptides revealed that their SMILES rather than their monomeric graph created the problem. The peptidic structures (SMILES) of these records contained errors or did not even correspond to the right molecule due to the ambiguous PubChem searches previously performed.

The eleven new monomers were added in the Norine database along with the correction of the wrong 


\section{a Curation of the SMILES in Norine database using rBAN}

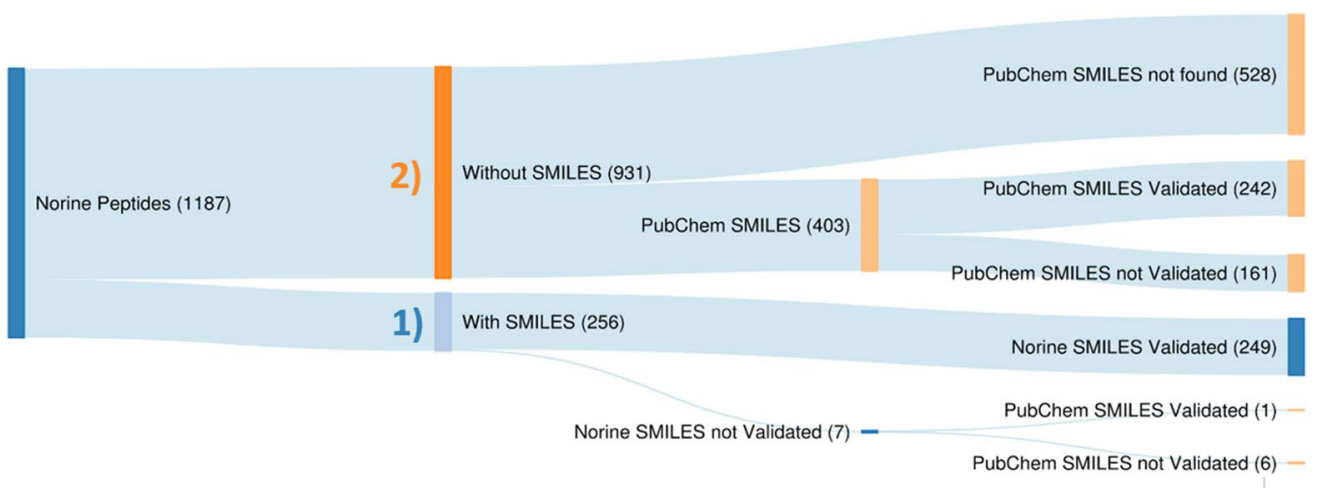

b Enniatin F - NOROO104

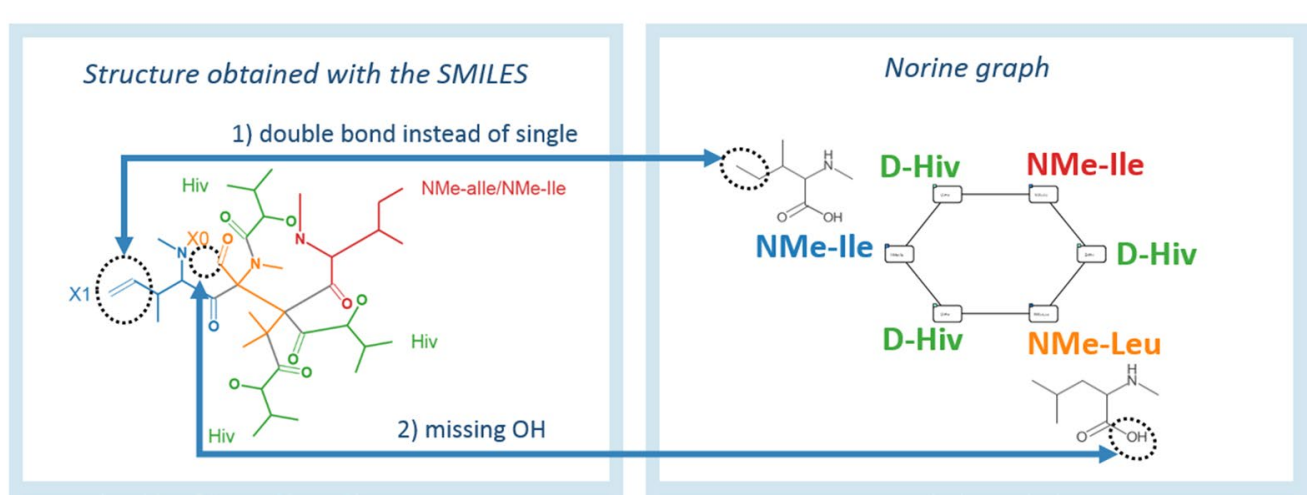

Fig. 5 Norine curation. a The curation involves two main steps: (1) Automatic verification and correction of the SMILES in Norine. rBAN validated 249 (97.26\%) SMILES and identified seven potential erroneous SMILES. Retrieving the PubChem SMILES from the non-validated entries enabled the correction of the SMILES of Motuporin (NOR00825). The manual inspection of the remaining entries concluded with the confirmation of six wrong SMILES. (2) Automatic addition of SMILES retrieved from PubChem. From the 403 SMILES retrieved from PubChem, 242 were validated using rBAN. The 161 not validated are likely to be false positives due to the ambiguity of the PubChem searches performed. $\mathbf{b}$ Enniatin $\mathrm{F}$ belongs to the set of non-validated peptides. rBAN failed to validate this peptide due to differences between the molecular and monomeric annotations. The monomeric graph is circular and contains N-Methyl-Isoleucine while the SMILES encodes a linear structure with dehydro-N-Methyl-Isoleucine(1). Additionally, rBAN could not identify what is supposed to be a N-Methyl-Leucine because it misses a hydroxyl group (2)

annotations, either in the monomeric graphs or in the SMILES of the compounds (find examples in Additional file 1: Fig. S1). This step was essential for evaluating the increase of the validated data. In the end, 11 added new monomers along with the correction of wrong annotations boosted the count from 492 to 526 validated entries.

\section{Benchmarking}

rBAN was compared against two tools with similar functionality: s2m and GRAPE. The benchmarking was performed on a PC computer with an Intel/Core i5-5300U CPU at $2.3 \mathrm{GHz}$ with $4 \mathrm{~GB}$ of RAM allocated to the Java Virtual Machine.

\section{rBAN vs s $2 m$}

Within the retro-biosynthetic tools targeting NRPs, s2m is the closest to rBAN as it produces the same output: a monomeric graph. Yet the two approaches substantially differ in their features and algorithmic approaches set to handle the issues raised by mapping the molecules. These involve among others, the monomer search, the light matching or the heterocycles treatment (see Table 2). In order to compare both tools, we analyzed their results, their robustness and their computational performance. The benchmark in the following sections was performed running $\mathrm{s} 2 \mathrm{~m}$ in the light matching mode to allow tautomer identification and obtain results comparable to those of rBAN.

Results comparison s2m was run to validate the same SMILES data previously used in the curation protocol of the Norine database. Out of the 659 peptidic structures retrieved from Norine and PubChem, s2m validated 445. Although the same process with rBAN resulted in a higher amount of validations (492), the comparison singled out 
Table 1 Monomers correctly suggested by rBAN

\begin{tabular}{|c|c|c|c|c|c|c|}
\hline Norine code & PubChemID & IUPAC name & Structure & Compounds & $\begin{array}{l}\text { Reason of the missing } \\
\text { monomer }\end{array}$ & Refs. \\
\hline NFo-Lys & 12679627 & $\begin{array}{l}\text { 6-amino-2-formami- } \\
\text { dohexanoic acid }\end{array}$ & & $\begin{array}{c}\text { NOR00261, NOR00262, } \\
\text { NOR00263, NOR00264 } \\
\text { NOR00266, NOR00267, } \\
\text { NOR00269, NOR00270 } \\
\text { NOR00271, NOR00272, } \\
\text { NOR00274, NOR00275 } \\
\text { NOR00276, NOR00277, } \\
\text { NOR00278, NOR00580 }\end{array}$ & "CO" monomer in graphs & {$[32]$} \\
\hline D-30Me-Ala & 97963 & $\begin{array}{l}\text { 2-amino-3-methoxypro- } \\
\text { panoic acid }\end{array}$ & & $\begin{array}{l}\text { NOR00422, NOR00423, } \\
\text { NOR00424, NOR00425 } \\
\text { NOR00588 }\end{array}$ & $\begin{array}{l}\text { Wrong SMILES of } \\
\text { D-3OMe-Ala monomer }\end{array}$ & [33] \\
\hline $\mathrm{C} 5: 1(4)-\mathrm{OH}(2)$ & 172026 & $\begin{array}{l}\text { 2-hydroxypent-4-enoic } \\
\text { acid }\end{array}$ & & $\begin{array}{l}\text { NOR00064, NOR00066, } \\
\text { NOR00068, NOR00071 } \\
\text { NOR00073 }\end{array}$ & $\begin{array}{l}\text { Wrong monomer in } \\
\text { graphs: } \mathrm{C} 4: 1(3)-\mathrm{OH}(2) \\
\text {-> C5:1(4)-OH(2) }\end{array}$ & {$[34]$} \\
\hline N-Suc & 12522 & $\begin{array}{l}\text { 4-amino-4-oxobutanoic } \\
\text { acid }\end{array}$ & & $\begin{array}{l}\text { NOR00160,NOR00166, } \\
\text { NOR00903 }\end{array}$ & $\begin{array}{l}\text { Missing monomer in } \\
\text { graphs }\end{array}$ & {$[35,36]$} \\
\hline $\mathrm{C} 5: 0-\mathrm{OH}(2)-\mathrm{Ep}(4)$ & 54305979 & $\begin{array}{l}\text { 2-hydroxy-3-(oxiran-2-yl) } \\
\text { propanoic acid }\end{array}$ & & NOR00086, NOR00087 & $\begin{array}{l}\text { Wrong monomer in } \\
\text { graphs: } \mathrm{C} 4: 0-\mathrm{OH}(2)- \\
\text { Ep(3) -> C5:0-OH(2)- } \\
\text { Ep(4) }\end{array}$ & {$[34]$} \\
\hline Gen & 3469 & $\begin{array}{l}\text { 2,5-dihydroxybenzoic } \\
\text { acid }\end{array}$ & & NOR00489, NOR00598 & $\begin{array}{l}\text { Wrong monomer in } \\
\text { graphs: 2,3-diOH-Bz } \\
\text {-> Gen }\end{array}$ & {$[37,38]$} \\
\hline $\mathrm{C} 10: 0-\mathrm{OH}(2)-\mathrm{NH} 2(3)$ & 57484230 & $\begin{array}{l}\text { 3-amino-2-hydroxydeca- } \\
\text { noic acid }\end{array}$ & & NOR01134, NOR01135 & $\begin{array}{l}\text { Wrong monomer in } \\
\text { graphs: Adda -> C10:0- } \\
\mathrm{OH}(2)-\mathrm{NH} 2(3)\end{array}$ & {$[39]$} \\
\hline iC6:0-OH(2.4) & 55300467 & $\begin{array}{l}\text { 2,4-dihydroxy-4-methyl- } \\
\text { pentanoic acid }\end{array}$ & & NOR00078, NOR00077 & $\begin{array}{l}\text { Wrong monomer in } \\
\text { graphs: } \mathrm{iC} 5: 0-\mathrm{OH}(2.3) \\
\text {-> iC6:0-OH(2.4) }\end{array}$ & {$[34]$} \\
\hline Isovaleric_acid & 10430 & 3-methylbutanoic acid & & NOR00477 & $\begin{array}{l}\text { Wrong monomer } \\
\text { in graph: Hiv -> } \\
\text { Isovaleric_acid }\end{array}$ & {$[40]$} \\
\hline D-Cl-Trp & 65259 & $\begin{array}{l}\text { 2-amino-3-(6-chloro-1H- } \\
\text { indol-3-yl)propanoic } \\
\text { acid }\end{array}$ & & NOR00554 & $\begin{array}{l}\text { Wrong SMILES of D-Cl- } \\
\text { Trp monomer }\end{array}$ & {$[41]$} \\
\hline
\end{tabular}

Among the suggested monomers, $\mathrm{N}$-Formyl-Lysine is the most abundant. rBAN considers $\mathrm{CO}$ as a formylation, therefore suggests a new formylated monomer instead of using the "CO" monomer currently present in Norine. A second new entity present in five compounds is D-3OMe-Ala. In this case the monomer name is correct but not the SMILES associated with it. Most of the other suggestions are due to the monomers wrongly annotated in the graph that should be substituted with a new substructure. There is also one case (N-Suc) where the monomer was directly missing in the graph. All these corrections were manually evaluated to confirm the agreement with the literature

five entries that were only verified by s2m (Fig. 6a). These entries were reviewed to identify the reasons why rBAN could not validate them. However, manual inspection only confirmed the validity of a single record, as the rest was not properly matching their monomeric counterparts and turned out to be false positives of s2m. Among these structures, Ennitatin F (Fig. 5b) that was reported earlier as cyclic and containing NMe-Leucine and NMeIsoleucine. $\mathrm{s} 2 \mathrm{~m}$ maps these monomers in the structure yet the NMe-Leucine is missing a hydroxyl group while the NMe-Isoleucine has an additional double bond. These artefacts are related to the method of precomputation and light matching in $\mathrm{s} 2 \mathrm{~m}$. Prior to the analysis, the precomputation of $\mathrm{s} 2 \mathrm{~m}$ generates for each monomer all the possible residues that may occur due to the loss of functional groups during the linkage with other monomers. These residues are the substructures that will be mapped by the software to identify the monomers. This strategy loses the association between the linkage and the loss of the functional group. That leads to wrong matches when the implicit hydrogens are not considered (as set in the light matching mode). This is the case of NMe-Leucine that is matched although it misses the hydroxyl group of the carboxyl end, which would be the expected structure if it was 
Table 2 Comparison rBAN versus s2m

\begin{tabular}{|c|c|c|}
\hline & rBAN & Smiles2Monomers \\
\hline a) Monomers mapping & $\begin{array}{l}\text { Based on molecule fragmentation through common } \\
\text { monomer linking bonds }\end{array}$ & $\begin{array}{l}\text { Based on mapping of monomers and selection of best } \\
\text { tiling }\end{array}$ \\
\hline b) Light matching & Positions of double/triple bonds are ignored & Implicit hydrogens and bond order are ignored \\
\hline c) Heterocycles treatment & $\begin{array}{l}\text { Accounts for NRP cyclisation patterns initiating oxazoles } \\
\text { and thiasoles formation }\end{array}$ & Does not include any rule/pattern for heterocycles \\
\hline d) Presence of new monomers & $\begin{array}{l}\text { Unmatched regions left unannotated and potentially } \\
\text { identified in discovery mode }\end{array}$ & $\begin{array}{l}\text { Matches the most similar monomers in a given database } \\
\text { and leaves out uncovered atoms }\end{array}$ \\
\hline e) Graph serialization & $\begin{array}{l}\text { Labelled edges with bond type and directed in accord- } \\
\text { ance to functional groups in each side }\end{array}$ & Unlabelled edges \\
\hline
\end{tabular}

a) To map the monomers rBAN fragments the molecule and matches the results against the monomer database. S2m computes the combinations of monomers that fit in the molecule. b) To enable tautomer identification during the matching process rBAN omits the positions of the double bonds in the monomer, but it keeps considering those, becoming more restrictive than its analog mode in $\mathrm{s} 2 \mathrm{~m}$, in which neither the implicit hydrogens nor the bonds order are taken into account. c) Characteristic NRP structural patterns such as heterocycles are specifically targeted in rBAN but not in s $2 \mathrm{~m}$. d) When a region cannot be matched because of the absence of the monomer in the database, rBAN leaves the whole region unannotated (with the option of recurring to the discovery mode), while $s 2 \mathrm{~m}$ tries to match the most similar monomer even if this is a wrong match and it implies leaving unannotated atoms. e) The monomers graph from rBAN has the edges labeled specifying the type of bond and its direction. $s 2 \mathrm{~m}$ does not provide bond labels

linked to another monomer, but is wrong when it is terminal in the molecule (see Additional file 1: Fig. S2). The three other false positives of $\mathrm{s} 2 \mathrm{~m}$ show similar problems. The fifth entry is Kermamide $K$, the only true positive in the set. It was not validated by $\mathrm{rBAN}$ because this software does not consider $\mathrm{CO}$ as a monomer.

The manual evaluation of the 52 peptides uniquely validated by rBAN confirmed their validity and uncovered some structural patterns that were optimally handled by rBAN and not by $\mathrm{s} 2 \mathrm{~m}$. The fragmentation model implemented in rBAN properly annotated large peptide structures whose monomeric composition was not revealed by the tiling algorithm of $\mathrm{s} 2 \mathrm{~m}$. Similarly, the annotation of NRPs containing thiazoles and oxazole heterocycles was successfully carried out using rBAN, while the results of $\mathrm{s} 2 \mathrm{~m}$ did not match the monomer graph. Another pattern also observed in the rBAN-validated entries was the presence of monomers whose hydrated and dehydrated forms coexist in the monomer database. The restrictive light matching of rBAN succeeded in distinguishing them, while the light matching approach of s2m led to wrong monomer assignments. Finally, to complete the picture of correctness, we computed the distribution of correctness values from each software (Fig. 6b). Both tools showed a similar distribution though slightly shifted. rBAN generates more highly scored peptides $(0.9-1)$ and less with correctness close to 0 .

Robustness comparison The existence of several combinations of monomers mapping the same peptide substructure increases the complexity of the problem. Hence, the extension of the monomer database can easily influence the mapping of a molecule and could lead to the appearance of wrong annotations that were previously correct. The robustness of the two software was tested while extending the monomer database and evaluating its impact on the results. An additional set of monomers was obtained using the PubChem Classification browser to retrieve the chemical entities defined as non-proteinogenic amino acids (ChEBI Ontology). Components with a molecular mass higher than $450 \mathrm{~g} / \mathrm{mol}$ were discarded, as they greatly exceeded the average monomer size. Chemical structures already present in the monomer database were also discarded to avoid repetitions. A total of 550 monomers were sequentially added in order to test the response of both software to different extensions of the database (Fig. 6c). rBAN correctly annotated the same amount of entries (492) for all the database sizes. Note that the number of correct annotations could not be improved because the Norine graphs were not modified to include the new monomers so maintaining the same correctness was the best that could be expected, proving the robustness of the software. On the other hand, s2m correct results dropped from 445 to 435 with the addition of 100 new monomers, although the rest of the extensions was steadily handled, only dropping by two in the extension to 450 monomers.

Computational performance comparison For the evaluation of the computational performance, the timing was limited to the analysis and did not account for the generation of images. We registered the performance of each software varying the number of input peptides from 100 to 600 . To obtain the average performance each measurement was repeated five times. rBAN was significantly faster than s2m (Fig. 6d). As expected, computing time increased with the number of peptides and the difference between the two software remained 4 and 5 -fold. Although this trend is likely to be confirmed, these measurements may change with a different set of peptides, as 


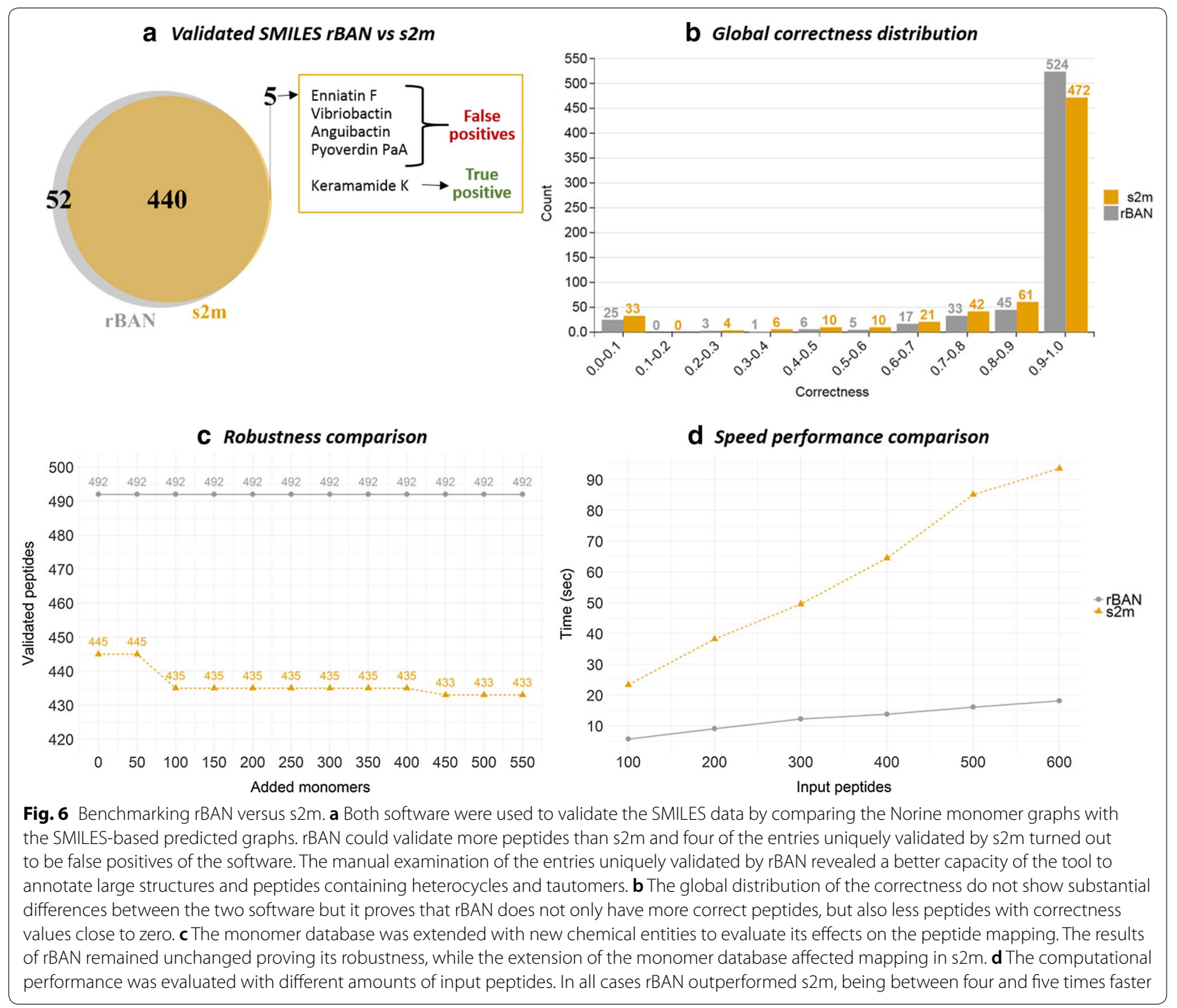

computing time depends on the complexity of the chemical structures analyzed. Note that with rBAN, using the discovery mode feature would also change the performance results as the computation time increases due to the RESTful HTTP requests performed to retrieve data from PubChem.

\section{rBAN vs GRAPE}

As already mentioned, GRAPE is another tool for the retro-biosynthesis of NRPs and polyketides (PKs). However, the annotations provided by this software differ from those of rBAN as (1) they are based on a different monomer library and (2) the modifications are annotated separately from the monomers. These differences make the comparison of correctness difficult and that explains why the benchmark was limited to the analysis of coverage (ratio between annotated atoms and total number of atoms in the molecule). The same set of SMILES without the wrong entries previously identified was used to test GRAPE. Out of 653 peptide structures, GRAPE fully annotated 468, while rBAN reaches 560 annotated entries, 492 of them being correct. In fact, from these results it is possible to indirectly compare the correctness of the two software. Only the peptides with a full coverage can have full correctness. Hence, assuming that all the annotations from GRAPE are correct (468), the result is still lower than the number of correctly annotated peptides from rBAN (492). The whole distribution of coverage shows how GRAPE tends to leave less peptides with low coverage (Fig. 7). Nevertheless, the annotations of the 18 peptides with zero coverage in rBAN were manually checked for GRAPE. As it turned out, their monomer fragments were categorized as "unknown". Finally, the computational performance was evaluated using the 
same data. rBAN analysed the 653 peptides in an average time of $26.94 \mathrm{~s}$, while the same process with GRAPE resulted in an average time of $81.34 \mathrm{~min}$.

Web implementation A web application interface was designed and integrated into Norine as an additional tool for the database curation. With the aim of providing a simple and user-friendly interface, the online version of rBAN is limited to the analysis of a single peptide. It only requires an input SMILES and it automatically depicts the peptide structure with the labeled monomers. Optionally, the Norine graph annotation can be introduced in order to obtain the graph correctness. The generated image can be downloaded in svg or png formats. Apart from the visual results, the serialized monomer graph is also provided as a json file. The discovery mode is still not available in the current web service version.

\section{Conclusions}

The usage of rBAN for Norine curation ended with the validation of $97 \%$ of the entries and the introduction of 242 SMILES and 11 monomers in the database. These results prove the ability of the algorithm to deduce effectively the monomeric graph of an NRP from its SMILES. The comparison with s2m and GRAPE clearly favored rBAN, which annotates more entries and with a higher perfomance. We also demonstrated the efficacy of the monomer discovery mode for the correction/addition of monomers. Furthermore, rBAN automatically produces graphs where the edges are labeled with the bond types linking the monomers. The current monomeric graphs in Norine do not contain this information, which is useful for the development of automatic substructure search. In the end, rBAN was integrated in Norine as a complementary tool for the future curation of the database. rBAN is accessible as a web service in Norine (http://bioinfo.crist al.univ-lille.fr/rban) and ExPASy (https://web.expasy.org/ rban). The jar is publicly available on bitbucket (https:// bitbucket.org/sib-pig/rban/downloads/).

\section{Limitations and perspectives}

The main limitation of the method is its dependence to the defined fragmentation rules. Hence, it fails mapping natural products following different patterns such as Polyketides (PKs). The introduction of new rules based on PK biosynthesis patterns would solve this issue and would extend the range of secondary metabolites covered. The software currently provides the results in a JSON format but returning the graphs in specific annotation formats such as HELM or SCSR is planned in order to improve the usability of the tool. Finally, the current slow performance of the discovery mode will be addressed by trying alternative programmatic access to PubChem data or by downloading a part of the PubChem database to our local server. In future versions of the software it would also be interesting to include a modification database and implement an optional mapping where the monomers and their modifications are annotated independently.

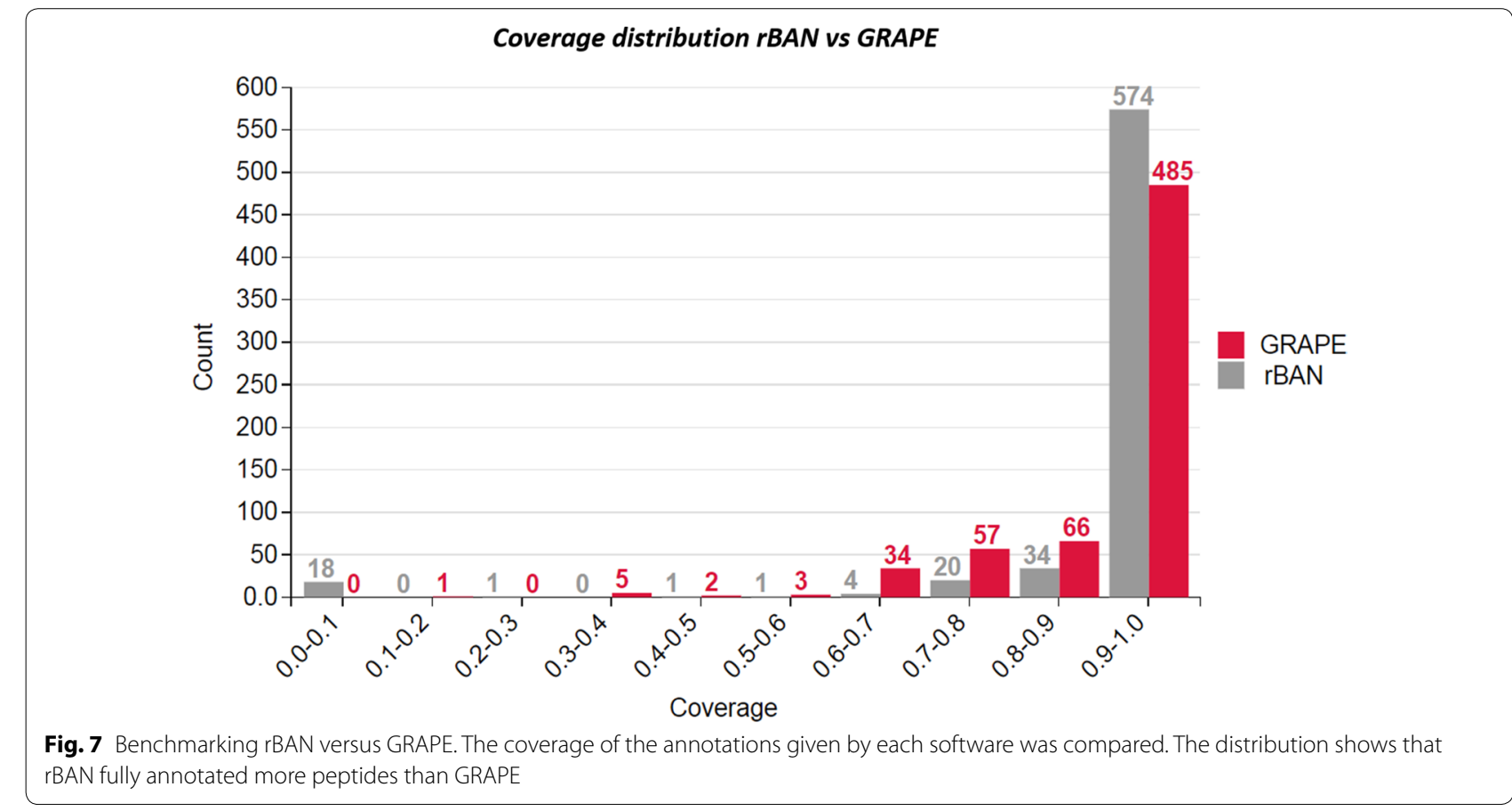




\section{Additional files}

Additional file 1. The file contains further details of the rBAN implementation and additional information of the analysis performed in the paper.

Additional file 2. Monomeric graph of Vancomycin. Example of a monomeric graph provided by rBAN.

\section{Authors' contributions}

Conceptualization, ER and FL; methodology, ER and MP; software, ER; validation, VL, AF, MP, MM; writing — original draft preparation, ER and FL; writingreview and editing, $M M, V L, M P, F L$; supervision, $M P$ and $F L$; funding acquisition, $\mathrm{VL}, \mathrm{MP}$ and $\mathrm{FL}$. All authors read and approved the final manuscript.

\section{Author details}

1 Proteome Informatics Group, SIB Swiss Institute of Bioinformatics, CMU, Rue Michel-Servet 1, 1211 Geneva, Switzerland. ${ }^{2}$ Computer Science Department, University of Geneva, Geneva, Switzerland. ${ }^{3}$ EA 7394-ICV- Institut Charles Viollette, University of Lille, INRA, ISA, University of Artois, Univ. Littoral Côte d'Opale, 59000 Lille, France. ${ }^{4}$ UMR 9189- CRIStAL-Centre de Recherche en Informatique Signal et Automatique de Lille, University of Lille, CNRS, Centrale Lille, 59000 Lille, France. ${ }^{5}$ Bonsai Team, Inria-Lille Nord Europe, 9655 Villeneuve d'Ascq Cedex, France. ${ }^{6}$ Vital-IT Group, SIB Swiss Institute of Bioinformatics, Amphipole Building, Quartier Sorge, 1015 Lausanne, Switzerland. ${ }^{7}$ Section of Biology, University of Geneva, Geneva, Switzerland.

\section{Acknowledgements}

We thank the IT group of the SIB Swiss Institute of Bioinformatics for their help on the integration of rBAN on the ExPASy server.

\section{Competing interests}

The authors declare that they have no competing interests.

\section{Availability of data and materials}

The interface of the software is available in the following webservers: https ://web.expasy.org/rban, http://bioinfo.cristal.univ-lille.fr/rban. A JAR file with more functionalities than the web application is available in Bitbucket: Project name: rBAN. Project home page: https://bitbucket.org/sib-pig/rban/downl oads. Archived version: BitBucket. Operating system(s): Platform independent. Programming language: Java. Other requirements: Java 1.8 or higher. License: Creative Commons Attribution-NonCommercial-ShareAlike 4.0 International Public License. The datasets supporting the conclusions of this article are included within the article (and its additional files).

\section{Funding}

SIB Fellowship programme and the European Union funding through the INTERREG Va FWVL SmartBioControl/Bioscreen Project.

\section{Publisher's Note}

Springer Nature remains neutral with regard to jurisdictional claims in published maps and institutional affiliations.

Received: 1 December 2018 Accepted: 31 January 2019

Published online: 08 February 2019

\section{References}

1. Newman DJ, Cragg GM (2016) Natural products as sources of new drugs from 1981 to 2014. J Nat Prod 79:629-661

2. Dejong CA, Chen GM, Li H et al (2016) Polyketide and nonribosomal peptide retro-biosynthesis and global gene cluster matching. Nat Chem Biol 12:1007

3. Medema MH, Blin K, Cimermancic P et al (2011) antiSMASH: rapid identification, annotation and analysis of secondary metabolite biosynthesis gene clusters in bacterial and fungal genome sequences. Nucleic Acids Res 39:W339-W346

4. Harwani D, Begani J, Lakhani J (2018) Genes to metabolites and metabolites to genes approaches to predict biosynthetic pathways in microbes for natural product discovery. In: Choudhary DK, Kumar M, Prasad R, Kumar V (eds) In silico approach for sustainable agriculture. Springer, Berlin, pp 1-16

5. Blin K, Kim HU, Medema MH, Weber T (2017) Recent development of antiSMASH and other computational approaches to mine secondary metabolite biosynthetic gene clusters. Brief Bioinform. https://doi. org/10.1093/bib/bbx146

6. Siani MA, Weininger D, Blaney JM (1994) CHUCKLES: a method for representing and searching peptide and peptoid sequences on both monomer and atomic levels. J Chem Inf Comput Sci 34:588-593

7. Lewell XQ, Judd DB, Watson SP, Hann MM (1998) Recap retrosynthetic combinatorial analysis procedure: a powerful new technique for identifying privileged molecular fragments with useful applications in combinatorial chemistry. J Chem Inf Comput Sci 38:511-522

8. Degen J, Wegscheid-Gerlach C, Zaliani A, Rarey M (2008) On the Art of Compiling and Using'Drug-Like'Chemical Fragment Spaces. ChemMedChem 3:1503-1507

9. Ghersi D, Singh M (2014) molBLOCKS: decomposing small molecule sets and uncovering enriched fragments. Bioinformatics 30:2081-2083

10. Dufresne $Y$, Noé L, Leclère $V$, Pupin M (2015) Smiles2Monomers: a link between chemical and biological structures for polymers. J Cheminform 7:62

11. Abdo A, Caboche $S$, Leclère $V$ et al (2012) A new fingerprint to predict nonribosomal peptides activity. J Comput Aided Mol Des 26:1187-1194

12. Caboche S, Pupin M, Leclère V et al (2009) Structural pattern matching of nonribosomal peptides. BMC Struct Biol 9:15

13. Caboche $S$, Leclère $V$, Pupin M et al (2010) Diversity of monomers in nonribosomal peptides: towards the prediction of origin and biological activity. J Bacteriol 192:5143-5150

14. Abdo A, Leclère $V$, Jacques $P$ et al (2014) Prediction of new bioactive molecules using a bayesian belief network. J Chem Inf Model 54:30-36

15. Zhang T, Li H, Xi H et al (2012) HELM: a hierarchical notation language for complex biomolecule structure representation. J Chem Inf Model 52:2796-2806. https://doi.org/10.1021/ci3001925

16. Milton J, Zhang T, Bellamy C et al (2017) HELM software for biopolymers. J Chem Inf Model 57:1233-1239

17. Chen WL, Leland BA, Durant JL et al (2011) Self-contained sequence representation: bridging the gap between bioinformatics and cheminformatics. J Chem Inf Model 51:2186-2208

18. Caboche $S$, Pupin $M$, Leclère $V$ et al (2007) NORINE: a database of nonribosomal peptides. Nucleic Acids Res 36:D326-D331

19. Flissi A, Dufresne Y, Michalik J et al (2015) Norine, the knowledgebase dedicated to non-ribosomal peptides, is now open to crowdsourcing Nucleic Acids Res 44:D1113-D1118

20. Dutta S, Dimitropoulos D, Feng Z et al (2014) Improving the representation of peptide-like inhibitor and antibiotic molecules in the Protein Data Bank. Biopolymers 101:659-668

21. Berman HM, Westbrook J, Feng Z et al (2000) The protein data bank. Nucleic Acids Res 28:235-242

22. Kim S, Thiessen PA, Bolton EE et al (2015) PubChem substance and compound databases. Nucleic Acids Res 44:D1202-D1213

23. Weininger $D$ (1988) SMILES, a chemical language and information system 1. Introduction to methodology and encoding rules. J Chem Inf Comput Sci 28:31-36

24. Felnagle EA, Jackson EE, Chan YA et al (2008) Nonribosomal peptide synthetases involved in the production of medically relevant natural products. Mol Pharm 5:191-211

25. Condurso HL, Bruner SD (2012) Structure and noncanonical chemistry of nonribosomal peptide biosynthetic machinery. Natural product reports 29:1099-1110

26. Giessen TW, Marahiel MA (2012) Ribosome-independent biosynthesis of biologically active peptides: application of synthetic biology to generate structural diversity. FEBS Lett 586:2065-2075

27. Bloudoff K, Schmeing TM (2017) Structural and functional aspects of the nonribosomal peptide synthetase condensation domain superfamily: discovery, dissection and diversity. Biochimica et Biophysica Acta (BBA)Proteins and Proteomics 1865:1587-1604 
28. Daylight Theory: SMARTS — a language for describing molecular patterns. http://www.daylight.com/dayhtml/doc/theory/theory.smarts.html. Accessed 29 Nov 2018

29. Walsh CT, Nolan EM (2008) Morphing peptide backbones into heterocycles. Proc Natl Acad Sci 105:5655-5656

30. Bloudoff K, Fage CD, Marahiel MA, Schmeing TM (2017) Structural and mutational analysis of the nonribosomal peptide synthetase heterocyclization domain provides insight into catalysis. Proc Natl Acad Sci 114:95-100

31. Crone WJK, Leeper FJ, Truman AW (2012) Identification and characterisation of the gene cluster for the anti-MRSA antibiotic bottromycin: expanding the biosynthetic diversity of ribosomal peptides. Chem Sci 3:3516-3521. https://doi.org/10.1039/C2SC21190D

32. Itou Y, Suzuki S, Ishida K, Murakami M (1999) Anabaenopeptins G and H, potent carboxypeptidase A inhibitors from the cyanobacterium Oscillatoria agardhii (NIES-595). Bioorg Med Chem Lett 9:1243-1246

33. Ford PW, Gustafson KR, McKee TC et al (1999) Papuamides A-D, HIV-inhibitory and cytotoxic depsipeptides from the sponges Theonella mirabilis and Theonella swinhoei collected in papua New Guinea. J Am Chem Soc 121:5899-5909

34. Pedras MSC, Zaharia LI, Ward DE (2002) The destruxins: synthesis, biosynthesis, biotransformation, and biological activity. Phytochemistry 59:579-596
35. Teintze M, Leong J (1981) Structure of pseudobactin A, a second siderophore from plant growth promoting Pseudomonas B10. Biochemistry 20:6457-6462

36. Atkinson RA, Salah El Din ALM, Kieffer B et al (1998) Bacterial iron transport: $1 \mathrm{H}$ NMR determination of the three-dimensional structure of the gallium complex of pyoverdin G4R, the peptidic siderophore of Pseudomonas putida G4R. Biochemistry 37:15965-15973

37. Chill L, Kashman Y, Schleyer M (1997) Oriamide, a new cytotoxic cyclic peptide containing a novel amino acid from the marine sponge Theonella sp. Tetrahedron 53:16147-16152

38. Fusetani N, Nakao Y, Matsunaga S (1991) Nazumamide A, a thrombininhibitory tetrapeptide, from a marine sponge, Theonella sp. Tetrahedron Lett 32:7073-7074

39. Sano T, Takagi H, Morrison LF et al (2005) Leucine aminopeptidase M inhibitors, cyanostatin A and B, isolated from cyanobacterial water blooms in Scotland. Phytochemistry 66:543-548

40. Nakao Y, Oku N, Matsunaga S, Fusetani N (1998) Cyclotheonamides E2 and $\mathrm{E} 3$, new potent serine protease inhibitors from the marine sponge of the genus Theonella. J Nat Prod 61:667-670

41. Schmidt EW, Faulkner DJ (1998) Microsclerodermins C-E, antifungal cyclic peptides from the lithistid marine sponges Theonella sp. and Microscleroderma sp. Tetrahedron 54:3043-3056
Ready to submit your research? Choose BMC and benefit from:

- fast, convenient online submission

- thorough peer review by experienced researchers in your field

- rapid publication on acceptance

- support for research data, including large and complex data types

- gold Open Access which fosters wider collaboration and increased citations

- maximum visibility for your research: over $100 \mathrm{M}$ website views per year

At BMC, research is always in progress.

Learn more biomedcentral.com/submissions 